\title{
VARIAÇÃO ESPAÇO-TEMPORAL DA DINÂMICA DA COMUNIDADE ARBÓREA EM FRAGMENTOS DE FLORESTA ALUVIAL EM MINAS GERAIS
}

\author{
Ana Carolina da Silva ${ }^{1}$, Pedro Higuchi², Eduardo van den Berg $^{3}$, Matheus Henrique Nunes ${ }^{4}$, \\ Marcela de Castro Nunes Santos ${ }^{5}$
}

(recebido: 6 de junho de 2010; aceito: 30 de junho de 2011)

\begin{abstract}
RESUMO: Neste estudo, objetivou-se conhecer os padrões de dinâmica da comunidade de árvores em fragmentos florestais aluviais nos períodos de 2005-2007 e 2007-2009. Para isso, foi estudado um fragmento de floresta ciliar e cinco fragmentos de floresta aluvial, no interior da planície de inundação do rio Sapucaí, em São Sebastião da Bela Vista, MG, inventariados, inicialmente, em 2005 e avaliados novamente em 2007 e em 2009. Os resultados demonstraram padrões diferenciados entre os fragmentos e os períodos de tempo. Nos fragmentos onde foi observada, em estudos anteriores, maior influência de enchentes e encharcamento do solo, a mortalidade de árvores foi maior no período de 2007-2009 do que no de 2005-2007. As taxas de mortalidade foram, de forma geral, maiores que as taxas de recrutamento, ocasionando a perda de indivíduos e a perda em área basal. Observando-se o histórico da área, é possível supor que essas perdas estão ocorrendo pela interação de dois fatores: i) excesso hídrico após uma grande enchente e ii) a ocorrência de forte antropização, representado pelo corte seletivo e a entrada de bovinos no interior dos fragmentos.
\end{abstract}

Palavras-chave: Dinâmica florestal, mata ciliar, fragmentos florestais.

\section{SPATIAL-TEMPORAL VARIATION IN THE DYNAMICS OF THE TREE COMMUNITY IN FRAGMENTS OF ALLUVIAL FOREST IN MINAS GERAIS}

\begin{abstract}
This study explored the dynamics pattern of the tree community in fragments of alluvial forest in the period 2005 2007 and in the period 2007-2009. The study comprises a fragment of riparian forest and five fragments of alluvial forest toward the interior of the floodplain of Sapucai river, in São Sebastião da Bela Vista, MG, initially inventoried in 2005 and then evaluated in 2007 and again in 2009. Results revealed different patterns among fragments and among time intervals. In the fragments where in previous studies a stronger influence of floods and sodden soil had been observed, tree mortality was found to be greater in the 2007-2009 period than in the 2005-2007 period. Overall, mortality rates were found to be higher than recruitment rates, leading to loss of individuals and loss of basal area. Judging by the history of the area, it can be assumed that these losses are due to an interaction of two factors: i) water excess after major flooding and ii) occurrence of strong anthropization, represented by selective logging and cattle introduction in the interior of the fragments.
\end{abstract}

Key words: Forest dynamics, riparian forest, forest fragments.

\section{INTRODUÇÃO}

Nas últimas décadas, houve um crescente interesse científico nos aspectos envolvidos na dinâmica florestal. Esse interesse tem sido ainda maior nos últimos anos, com a necessidade de se entender a influência de ecossistemas florestais sobre o clima, principalmente em decorrência do fato de as árvores poderem sequestrar ou emitir carbono para a atmosfera, por meio do acúmulo, que ocorre durante o seu crescimento, ou perda de biomassa, em decorrência de eventos naturais ou antrópicos. Porém, por tais estudos serem recentes e muitas espécies florestais apresentarem elevada longevidade, algumas milenares (CHAMBERS et al., 1998), o conhecimento sobre os processos de dinâmica de florestas, principalmente em florestas tropicais nativas, ainda é limitado. Além disso, a existência de uma elevada diversidade alfa e beta de espécies (CONDIT et al., 2002) sugere que a generalização dos resultados encontrados para ambientes distintos não seja adequada.

\footnotetext{
${ }^{1}$ Engenheira Florestal, Professora Dra. em Engenharia Florestal - Departamento de Engenharia Florestal - Universidade do Estado de Santa Catarina Av. Luiz de Camões, 2090 - B. Conta Dinheiro - 88520-000 - Lages, SC - carol_sil4@yahoo.com.br

${ }^{2}$ Engenheiro Florestal, Professor Dr. em Engenharia Florestal - Departamento de Engenharia Florestal - Universidade do Estado de Santa Catarina Av. Luiz de Camões, 2090 - B. Conta Dinheiro - 88520-000 - Lages, SC - higuchip@gmail.com

${ }^{3}$ Agrônomo, Professor Dr. em Biologia Vegetal - Departamento de Biologia - Universidade Federal de Lavras - 37200-000 - Lavras, MG evandenb@dbi.ufla.br

${ }^{4}$ Engenheiro Florestal, Mestrando em Recursos Florestais - Departamento de Ciências Florestais - ESALQ - Universidade de São Paulo - Av. Pádua Dias, 11 - 13418-900 - Piracicaba, SP - matheusfloresta.ptc@hotmail.com

${ }^{5}$ Engenheira Florestal, Mestranda em Engenharia Florestal - Universidade Federal de Lavras - Departamento de Ciências Florestais - 37200-000 Lavras, MG-marcelacns@gmail.com
}

Cerne, Lavras, v. 17, n. 4, p. 465-471, out./dez. 2011 
Estudos da dinâmica florestal em florestas inundáveis são ainda mais raros, podendo-se destacar, no centro-sul do Brasil, os estudos de Appolinário et al. (2005), Damasceno Júnior et al. (2004), Guilherme et al. (2004), Guimarães et al. (2008) e Silva et al. (2011a). Esses estudos são importantes, pois permitem o monitoramento e a previsão dos processos de transformação das populações e comunidades vegetais (SHEIL et al., 2000), além de servir para a realização de inferências sobre a possibilidade de a floresta estar funcionando como sumidouro ou fonte de carbono para atmosfera. Os dados obtidos também permitem o subsídio de estratégias de manejo, visando ao uso sustentável dos recursos florestais e a restauração florestal.

Dessa forma, neste trabalho, objetivou-se conhecer os padrões de dinâmica da comunidade arbórea em fragmentos florestais aluviais nos períodos de 2005-2007 e 2007-2009, além de testar as seguintes hipóteses: i) As taxas de dinâmica apresentam variações entre os períodos estudados; e ii) a dinâmica apresenta variação espacial refletindo a heterogeneidade ambiental existente.

\section{MATERIAL E MÉTODOS}

\subsection{Descrição da área de estudo}

A área está localizada no município de São Sebastião da Bela Vista, MG, onde foram estudados seis fragmentos florestais, sendo um fragmento de floresta ciliar sobre o dique natural adjacente ao Rio Sapucaí e cinco fragmentos florestais na planície aluvial localizados após o dique (Figura 1). As coordenadas geográficas da área estão nos limites de latitude $22^{\circ} 05^{\prime} 57^{\prime \prime} \mathrm{S}$ a $22^{\circ} 07^{\prime} 22,5^{\prime \prime} \mathrm{S}$ e longitude $45^{\circ} 48^{\prime} 05^{\prime \prime} \mathrm{W}$ a $45^{\circ} 48^{\prime} 53,5^{\prime \prime} \mathrm{W}$, sendo altitude média de $809 \mathrm{~m}$. A área dos fragmentos estudados são: 8,15 ha (floresta ciliar), 7,47 ha (fragmento 1), 21,69 ha (fragmento 2), 2,00 ha (fragmento 3), 1,62 ha (fragmento 4) e 0,90 ha (fragmento 5). Segundo a classificação da Empresa Brasileira de Pesquisa Agropecuária - Embrapa (1999), os solos da região são, predominantemente, Neossolos, na floresta ciliar, Gleissolos, nas áreas de maior encharcamento e Cambissolos, nas áreas menos saturadas (SILVA et al., 2009). Segundo a classificação de Köppen, o clima predominante na região é mesotérmico úmido, com dois a três meses secos ao ano, enquadrado no tipo Cwb (BRASIL, 1992). As florestas da área de estudos são classificadas como Floresta Estacional Semidecidual Aluvial (INSTITUTO BRASILEIRO DE GEOGRAFIA E ESTATÍSTICA - IBGE, 1992). Os fragmentos aluviais foram inventariados no ano de 2005 (SILVA et al., 2009)

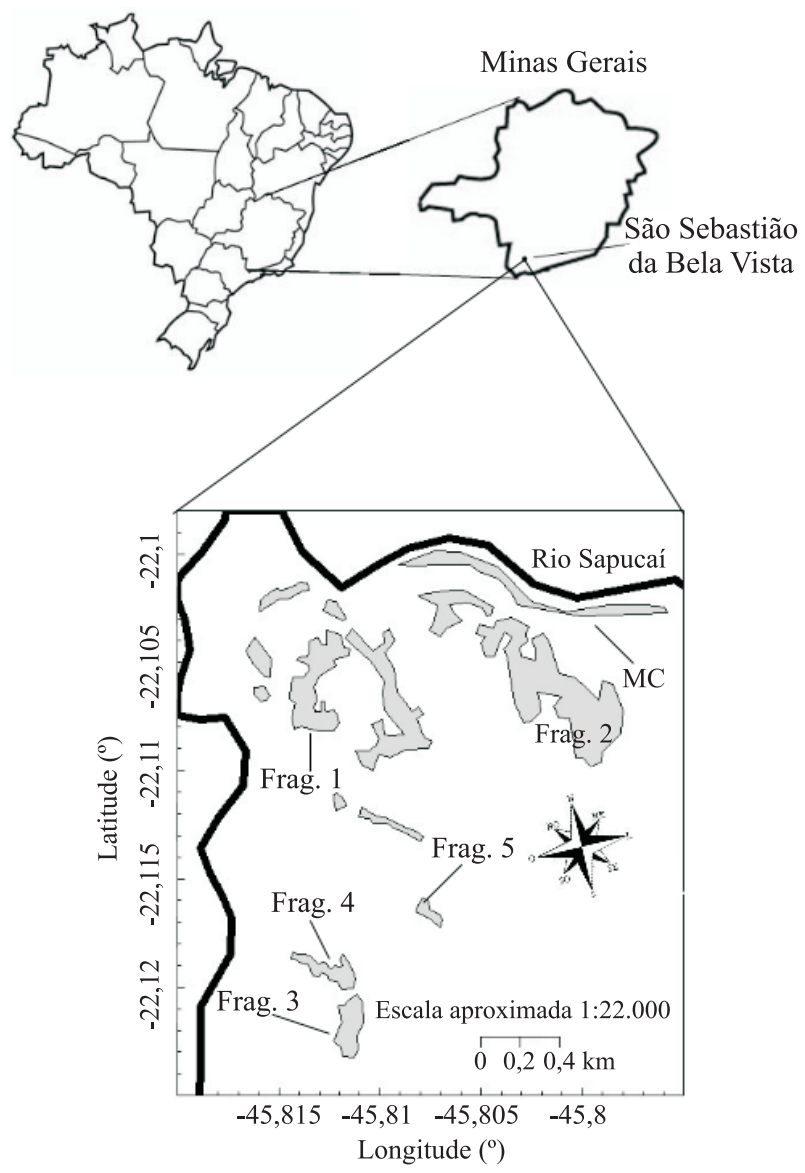

Figura 1 - Fragmento de Mata ciliar (MC) e demais fragmentos florestais estudados no Município de São Sebastião da Bela Vista, Minas Gerais, Brasil. O rio Sapucaí está representado em preto, os fragmentos florestais presentes na área estão em cinza e, em branco, a matriz não florestal. Fonte: Silva et al. (2009).

Figure 1 - Fragment of riparian forest (MC) and other forest fragments studied in the municipality of São Sebastião da Bela Vista, Minas Gerais, Brazil. Sapucai River is depicted in black, forest fragments are depicted in grey, and the nonforest matrix, in white. Source: Silva et al. (2009).

e sua dinâmica foi primeiramente avaliada para o intervalo de 2005-2007 (SILVA et al., 2011a). Foi registrada uma forte enchente ocorrida em janeiro de 2007, onde as árvores da floresta ciliar e dos fragmentos 1, 3, 4 e 5 ficaram parcialmente submersas (SILVA et al., 2011a). Segundo os mesmos autores, a floresta ciliar foi afetada pela correnteza da enchente por estar próxima à calha principal do rio, ao contrário dos fragmentos, onde houve apenas o aumento do nível freático, porém, com encharcamento de maior duração que na floresta ciliar.

Cerne, Lavras, v. 17, n. 4, p. 465-471, out./dez. 2011 


\subsection{Amostragem da vegetação}

Os estudos de dinâmica foram conduzidos nas 54 parcelas de $200 \mathrm{~m}^{2}(10 \times 20 \mathrm{~m})$, alocadas em 2005 (SILVA et al., 2009). Destas, 24 parcelas foram alocadas na floresta ciliar e seis parcelas em cada um dos cinco fragmentos aluviais estudados. No primeiro inventário, todos os indivíduos arbóreos vivos dentro das parcelas que apresentaram diâmetro medido a 1,30 m de altura (DAP) igual ou superior a $5 \mathrm{~cm}$ foram identificados, etiquetados e mensurados (DAP e altura) no mês de julho de 2005, sendo que indivíduos com caules múltiplos foram medidos quando a soma das áreas basais das secções dos caules correspondia a uma área basal igual ou maior que a de um caule único com $5 \mathrm{~cm}$ de DAP. As identificações foram realizadas por meio de consultas a especialistas, literatura e herbários, sendo as espécies classificadas nas famílias reconhecidas pelo sistema APG III (ANGIOSPERM PHYLOGENY GROUP - APG, 2009). No mês de julho de 2007, dois anos após o primeiro inventário, foi realizado o segundo levantamento da comunidade arbórea (SILVA et al., 2011a), e no mês de julho de 2009, após dois anos do segundo inventário, foi realizado o terceiro inventário. Para ambos, utilizou-se a mesma metodologia descrita anteriormente. Os indivíduos recrutas que atingiram o DAP $\geq 5 \mathrm{~cm}$ foram etiquetados, identificados e medidos. Os indivíduos mortos foram registrados e os sobreviventes de cada inventário anterior, mensurados novamente.

\subsection{Dinâmica da comunidade arbórea}

Foram calculadas para a amostra total, para as amostras da floresta ciliar e de cada fragmento as taxas de mortalidade e recrutamento, as taxas de ganho e perda em área basal, com base no número de indivíduos e área basal, por meio dos modelos algébricos citados por Sheil e May (1996). A taxa média anual de rotatividade em número de árvores (Rotn) foi calculada pela média das taxas de mortalidade e de recrutamento e a taxa média anual de rotatividade em área basal (Rotab) foi calculada pela média de taxa de perda e taxa de ganho (KORNING; BALSLEV, 1994; OLIVEIRA FILHO et al., 1997).

Para avaliar se existem diferenças entre os períodos de tempo estudados (2005-2007 e 2007-2009) em relação ao número de indivíduos mortos e recrutados e à área basal dos indivíduos mortos, recrutados e sobreviventes, foi utilizado o teste de Mann-Whitney. Para verificar a variação espacial das taxas de dinâmica da comunidade arbórea, foram comparadas as taxas de mortalidade, recrutamento, ganho e perda em área basal e rotatividade em número de indivíduos e em área basal, entre a floresta ciliar e os demais fragmentos estudados, pelas análises de variância não paramétrica de KruskalWallis. Todas as análises foram realizadas no programa $\mathrm{R}$ (R DEVELOPMENT CORE TEAM, 2009).

\section{RESULTADOS}

No fragmento de floresta ciliar, o número de indivíduos mortos no período 2007-2009 (97,9 ind ha-1) superou a mortalidade no período 2005-2007 (45,8 ind ha-1) (Tabela 1). Esse padrão foi seguido por outros fragmentos diretamente afetados pelas enchentes, como pelo fragmento 1, que apresentou 191,7 ind ha ${ }^{-1}$ mortos em 2007-2009 e somente 50,0 ind ha ${ }^{-1}$ mortos no período 2005-2007 e pelo fragmento 3, que obteve 275, 0 ind ha $^{-1}$ mortos em 2007-2009 e 141,7 ind ha-1 mortos em 20052007. O mesmo padrão não foi seguido pelos fragmentos 2 (50,0 ind ha ${ }^{-1}$ mortos em ambos os períodos), 4 (233,3 ind ha-1 mortos no período 2005-2007 e 225,0 ind ha-1 mortos no período 2007-2009) e 5 (25,0 ind ha ${ }^{-1}$ mortos no período 2005-2007 e 91,7 ind ha-1 no período 20072009).

A maior taxa de mortalidade no período 2005-2007 foi no fragmento $4\left(5,06 \% \mathrm{ano}^{-1}\right)$. No período 2007-2009 não houve diferenças significativas entre os fragmentos para a taxa de mortalidade. Influenciada, principalmente, pela alta mortalidade, a taxa de rotatividade também foi alta, destacando-se a taxa observada no fragmento 4 no período de 2005-2007 $\left(3,51 \%\right.$ ano $\left.^{-1}\right)$.

No período 2005-2007, todos os fragmentos apresentaram taxa de recrutamento menor do que a taxa de mortalidade (SILVA et al., 2011a). No período 2007-2009, houve padrão semelhante, com, somente, o fragmento 2 apresentando taxa de recrutamento $\left(2,97 \%\right.$ ano $\left.^{-1}\right)$ maior que a taxa de mortalidade $\left(1,61 \%\right.$ ano $\left.^{-1}\right)$.

Assim, houve diminuição do número de árvores na área total estudada de 2062 (em 2005) para 1930 (em 2009), com a perda de 132 árvores. Somada à diminuição de número de indivíduos, houve também decréscimo de área basal, que passou de 44,86 $\mathrm{m}^{2}$ em 2005 para 43,12 $\mathrm{m}^{2}$ em 2009, resultando na diminuição de $1,74 \mathrm{~m}^{2}$ nesses quatro anos, considerando todas as áreas e períodos. Não houve diferenças significativas entre as taxas de perda em área basal dos fragmentos em ambos os períodos (Tabela 2).

Cerne, Lavras, v. 17, n. 4, p. 465-471, out./dez. 2011 
Tabela 1 - Dinâmica da comunidade arbórea, nos períodos de 2005-2007 e 2007-2009, em fragmentos de floresta aluvial em São Sebastião da Bela Vista, MG, para a floresta ciliar (FC) e os demais fragmentos estudados (Frag 1, 2, 3, 4 e 5), expressa em número de árvores por hectare.

Table 1-Dynamics of the tree community, in the 2005-2007 period and in the 2007-2009 period, in fragments of alluvial forest in São Sebastião da Bela Vista, $M G$, for riparian forest (FC) and other fragments studied (Frag 1, 2, 3, 4 and 5), expressed as number of trees per hectare.

\begin{tabular}{|c|c|c|c|c|c|c|c|}
\hline Períodos & & $\mathrm{FC}$ & Frag 1 & Frag 2 & Frag 3 & Frag 4 & Frag 5 \\
\hline & Número de indivíduos em 2005 (ind ha-1) & 1356,3 & 2350,0 & 1575,0 & 3125,0 & 2366,7 & 2341,7 \\
\hline \multirow{7}{*}{$2005-2007$} & Mortos (ind ha-1) & $45,8^{+}$ & $50,0^{+}$ & 50,0 & $141,7^{+}$ & 233,3 & 25,0 \\
\hline & Recrutas (ind ha-1) & 29,2 & 33,3 & 41,7 & 41,7 & 91,7 & $8,33^{+}$ \\
\hline & Sobreviventes (ind ha ${ }^{-1}$ ) & 1310,4 & 2300,0 & 1525,0 & 2983,3 & 2133,3 & 2316,7 \\
\hline & Taxa de mortalidade $\left(\%\right.$ ano $\left.^{-1}\right)$ & $1,70^{*}$ & $1,07^{*}$ & $1,60^{*}$ & $2,29^{*}$ & $5,06^{*}$ & $0,54^{*}$ \\
\hline & Taxa de recrutamento $\left(\%\right.$ ano $\left.^{-1}\right)$ & $1,08^{\mathrm{ns}}$ & $0,71^{\mathrm{ns}}$ & $1,33^{\mathrm{ns}}$ & $0,67^{\mathrm{ns}}$ & $1,96^{\mathrm{ns}}$ & $0,18^{\mathrm{ns}}$ \\
\hline & Taxa de rotatividade $\left(\% \mathrm{ano}^{-1}\right)$ & $1,39^{*}$ & $0,89^{*}$ & $1,47^{*}$ & $1,48^{*}$ & $3,51^{*}$ & $0,36^{*}$ \\
\hline & Número de indivíduos em 2007 (ind ha-1) & 1339,6 & 2333,3 & 1566,7 & 3025,0 & 2225,0 & 2325,0 \\
\hline \multirow{7}{*}{ 2007-2009 } & Mortos (ind ha-1) & $97,9^{+}$ & $191,7^{+}$ & 50,0 & $275,0^{+}$ & 225,0 & 91,7 \\
\hline & Recrutas (ind ha ${ }^{-1}$ ) & 37,5 & 25,0 & 91,7 & 58,3 & 100,0 & $50,0^{+}$ \\
\hline & Sobreviventes (ind ha ${ }^{-1}$ ) & 1241,7 & 2141,7 & 1516,7 & 2750,0 & 2000,0 & 2233,3 \\
\hline & Taxa de mortalidade $\left(\%\right.$ ano $\left.^{-1}\right)$ & $3,72^{\mathrm{ns}}$ & $4,20^{\mathrm{ns}}$ & $1,61^{\mathrm{ns}}$ & $4,65^{\mathrm{ns}}$ & $5,19^{\text {ns }}$ & $1,99^{\text {ns }}$ \\
\hline & Taxa de recrutamento $\left(\%\right.$ ano $\left.^{-1}\right)$ & $1,41^{\mathrm{ns}}$ & $0,54^{\mathrm{ns}}$ & $2,97^{\mathrm{ns}}$ & $0,97^{\mathrm{ns}}$ & $2,27^{\mathrm{ns}}$ & $1,08^{\mathrm{ns}}$ \\
\hline & Taxa de rotatividade $\left(\%\right.$ ano $\left.^{-1}\right)$ & $2,57^{\mathrm{ns}}$ & $2,37^{\mathrm{ns}}$ & $2,29^{\text {ns }}$ & $2,81^{\mathrm{ns}}$ & $3,73^{\text {ns }}$ & $1,54^{\mathrm{ns}}$ \\
\hline & Número de indivíduos em 2009 (ind ha-1) & 1279,2 & 2166,7 & 1608,3 & 2808,3 & 2100,0 & 2283,3 \\
\hline
\end{tabular}

${ }^{*}$ Diferença significativa entre os fragmentos $(p<0,05)$ pelo teste de Kruskal-Wallis; ${ }^{\text {ns }}$ não há diferença significativa a $5 \%$ de probabilidade entre os fragmentos pelo teste de Kruskal-Wallis. ${ }^{+}$Representa diferença significativa $(p<0,10)$ entre os períodos de tempo pelo teste de Mann-Whitney.

A taxa de ganho em área basal foi maior nos fragmentos 3 e 4, em ambos os períodos (2005-2007, $3,27 \%$ ano $^{-1}$ e $3,72 \%$ ano $^{-1}$, respectivamente, e $2007-$ $2009,5,62 \%$ ano $^{-1}$ e $5,53 \%$ ano $^{-1}$, respectivamente) e foi influenciada, principalmente, pelo crescimento dos sobreviventes, já que o recrutamento foi pequeno. Porém, ao contrário desses fragmentos onde o crescimento das sobreviventes contribuiu para o aumento da área basal, na floresta ciliar, houve perda no período 2007-2009 $\left(-0,25 \mathrm{~m}^{2} \mathrm{ha}^{-1}\right)$. As altas taxas de ganho e de perda em área basal ocasionaram, nos fragmentos 3 e 4 , no período 2005-2007, as maiores taxas de rotatividade em área basal. No período 2007-2009, não houve diferença significativa entre os fragmentos em taxa de rotatividade em área basal.

\section{DISCUSSÃO}

A dinâmica da comunidade arbórea apresentou variação espacial e entre os períodos avaliados de 20052007 e 2007-2009. Assim, provavelmente, a dinâmica está respondendo às variações existentes entre os fragmentos em termos ambientais, florísticos e estruturais constatadas em estudos anteriores (SILVA et al., 2009, 2011c). Outros trabalhos têm apontado que taxas de dinâmica não são homogêneas em fragmentos florestais do Alto Rio Grande, variando espacialmente dentro das áreas (HIGUCHI et al., 2008; OLIVEIRA-FILHO et al., 2007).

A intensidade das enchentes parece ter sido o principal fator influenciador das taxas de dinâmica. Nos fragmentos onde a enchente de maior proporção ocorrida em janeiro de 2007 foi mais intensa (floresta ciliar e fragmentos 1,3 e 5), as taxas de mortalidade tenderam a ser maiores que as de recrutamento. Já, no fragmento 2, o menos afetado pela inundação, as taxas de mudança referentes ao número de indivíduos e à área basal foram próximas a zero. Segundo Damasceno Júnior et al. (2004), enchentes mais intensas ocasionam maior velocidade da água do rio, maior taxa de sedimentação e maior remoção de substrato no barranco do dique, fatores que causam maior tombamento de árvores e, consequentemente,

Cerne, Lavras, v. 17, n. 4, p. 465-471, out./dez. 2011 
Tabela 2 - Dinâmica da comunidade arbórea, nos períodos de 2005-2007 e 2007-2009, em fragmentos de floresta aluvial em São Sebastião da Bela Vista, MG, para a floresta ciliar (MC) e os demais fragmentos estudados (Frag 1, 2, 3, 4 e 5), expressa em área basal.

Table 2 - Dynamics of the tree community in the 2005-2007 period and in the 2007-2009 period, in fragments of alluvial forest in São Sebastião da Bela Vista, MG, for riparian forest (MC) and other fragments studied (Frag 1, 2, 3, 4 and 5), expressed as basal area.

\begin{tabular}{|c|c|c|c|c|c|c|c|}
\hline Períodos & & $\mathrm{FC}$ & Frag 1 & Frag 2 & Frag 3 & Frag 4 & Frag 5 \\
\hline & Área basal (AB) em $2005\left(\mathrm{~m}^{2} \mathrm{ha}^{-1}\right)$ & 46,04 & 41,42 & 34,08 & 35,42 & 27,58 & 51,17 \\
\hline \multirow{7}{*}{ 2005-2007 } & $\mathrm{AB}$ das mortas $\left(\mathrm{m}^{2} \mathrm{ha}^{-1}\right)$ & $1,10^{+}$ & $0,58^{+}$ & 0,50 & 0,92 & 1,92 & 0,25 \\
\hline & $\mathrm{AB}$ das recrutas $\left(\mathrm{m}^{2} \mathrm{ha}^{-1}\right)$ & 0,06 & 0,08 & $0,10^{+}$ & 0,11 & 0,23 & 0,025 \\
\hline & Crescimento das sobreviventes $\left(\mathrm{m}^{2} \mathrm{ha}^{-1}\right)$ & 0,35 & 0,75 & 0,33 & $0,17^{+}$ & 0,92 & 0,33 \\
\hline & Taxa de ganho $\left(\%\right.$ ano $\left.^{-1}\right)$ & $2,15^{* *}$ & $2,00^{* *}$ & $2,10^{* *}$ & $3,27^{* *}$ & $3,72^{* *}$ & $2,03^{* *}$ \\
\hline & Taxa de perda $\left(\%\right.$ ano $\left.^{-1}\right)$ & $2,86^{\mathrm{ns}}$ & $1,77^{\mathrm{ns}}$ & $2,13^{\mathrm{ns}}$ & $4,07^{\mathrm{ns}}$ & $4,99^{\mathrm{ns}}$ & $1,93^{\text {ns }}$ \\
\hline & Taxa de rotatividade $\left(\%\right.$ ano $\left.^{-1}\right)$ & $2,51^{* *}$ & $1,88^{* *}$ & $2,11^{* *}$ & $3,67^{* *}$ & $4,36^{* *}$ & $1,98^{* *}$ \\
\hline & AB em $2007\left(\mathrm{~m}^{2} \mathrm{ha}^{-1}\right)$ & 45,38 & 41,67 & 34,08 & 34,83 & 26,83 & 51,25 \\
\hline \multirow{7}{*}{ 2007-2009 } & $\mathrm{AB}$ das mortas $\left(\mathrm{m}^{2} \mathrm{ha}^{-1}\right)$ & $2,15^{+}$ & $4,25^{+}$ & 0,50 & 1,92 & 2,33 & 0,58 \\
\hline & $\mathrm{AB}$ das recrutas $\left(\mathrm{m}^{2} \mathrm{ha}^{-1}\right)$ & 0,21 & 0,06 & $0,23^{+}$ & 0,15 & 0,24 & 0,11 \\
\hline & Crescimento das sobreviventes $\left(\mathrm{m}^{2} \mathrm{ha}^{-1}\right)$ & $-0,25$ & 1,08 & 0,50 & $2,58^{+}$ & 1,58 & 1,08 \\
\hline & Taxa de ganho $\left(\%\right.$ ano $\left.^{-1}\right)$ & $2,68^{* *}$ & $2,96^{* *}$ & $2,55^{* *}$ & $5,62^{* *}$ & $5,53^{* *}$ & $3,04^{* *}$ \\
\hline & Taxa de perda $\left(\%\right.$ ano $\left.^{-1}\right)$ & $5,08^{\mathrm{ns}}$ & $6,70^{\mathrm{ns}}$ & $2,15^{\mathrm{ns}}$ & $4,44^{\mathrm{ns}}$ & $6,51^{\mathrm{ns}}$ & $2,47^{\mathrm{ns}}$ \\
\hline & Taxa de rotatividade $\left(\%\right.$ ano $\left.^{-1}\right)$ & $3,90^{\text {ns }}$ & $4,83^{\mathrm{ns}}$ & $2,35^{\text {ns }}$ & $5,03^{\mathrm{ns}}$ & $6,02^{\mathrm{ns}}$ & $2,76^{\mathrm{ns}}$ \\
\hline & AB em $2009\left(\mathrm{~m}^{2} \mathrm{ha}^{-1}\right)$ & 43,19 & 38,50 & 34,33 & 35,67 & 26,25 & 51,83 \\
\hline
\end{tabular}

${ }^{* *}$ Diferença significativa entre os fragmentos $(p<0,01)$ pelo teste de Kruskal-Wallis; ${ }^{\text {ns }}$ não há diferença significativa a $5 \%$ de probabilidade entre os fragmentos pelo teste de Kruskal-Wallis. ${ }^{+}$Representa diferença significativa $(p<0,10)$ entre os períodos de tempo pelo teste de Mann-Whitney.

maior mortalidade. Já, a baixa taxa de recrutamento em florestas inundáveis é explicada por autores que consideram que a deposição de sedimentos pelas enchentes limita o estabelecimento das plantas (JUNK, 1993), além de causarem limitações para o crescimento, devido pela obstrução da captura de luz e trocas gasosas (APPOLINÁRIO et al., 2005), o que poderia limitar o recrutamento no nível de inclusão estudado. Os resultados demonstram que, ao contrário do fragmento 2, pouco influenciado pelas enchentes na região, os fragmentos estão em processo de degeneração pós-distúrbio.

A maior mortalidade encontrada no segundo período analisado quando comparado com o primeiro, nos fragmentos mais influenciados pela enchente, aponta para um efeito retardado da enchente sobre a sobrevivência das árvores. A primeira avaliação da dinâmica ocorreu apenas seis meses após a enchente e, aparentemente, não computou a totalidade do seu efeito sobre a comunidade, algo só percebido no terceiro inventário. Franklin et al. (1987) salienta que a mortalidade de árvores pode ser lenta. Ela envolve, primeiramente, um estresse fisiológico, posteriormente uma fase de senescência e, finalmente, a morte. Muitas árvores vivas avaliadas no período 2005-2007 estavam em processo de senescência ou quebradas e, cerca de dois terços destas foram encontradas, posteriormente, mortas na avaliação do período 2007-2009. O fragmento 2 não seguiu o mesmo padrão (mesmo número de mortos em ambos os períodos), porque, conforme já comentado, é o menos influenciado pelas enchentes.

Além da enchente, secundariamente outros fatores podem estar influenciando as taxas de dinâmica. O fragmento 4 tem sido fortemente influenciado por impactos antrópicos ao longo dos anos, anteriores e posteriores ao primeiro levantamento em 2005, com a ocorrência de cortes seletivos e penetração de gado constantemente, resultando em elevada mortalidade no primeiro período avaliado. Isso não quer dizer que as taxas de mortalidade não tenham sido influenciadas ali também pelas enchentes, mas não é possível separar o efeito do impacto antrópico do resultado da enchente.

Cerne, Lavras, v. 17, n. 4, p. 465-471, out./dez. 2011 
Por esses fatores, considerando-se todas as áreas e períodos em que se constatou a perda de 132 árvores e de $1,74 \mathrm{~m}^{2}$ em área basal (em 1,08 ha estudados), sugerese que há instabilidade na dinâmica dos fragmentos estudados. O padrão esperado na dinâmica de florestas não perturbadas em avançado estágio de sucessão é de equilíbrio entre as taxas de ganho/perda e recrutamento/ mortalidade (LIEBERMAN et al., 1985; SWAINE et al., 1987).

Apesar da predominância de perda em área basal, verificou-se que os fragmentos 3 e 4 destacaramse, em ambos os períodos, com as mais altas taxas de ganho em área basal. Isso pode ser um reflexo da alta taxa de mortalidade que vem ocorrendo nesses fragmentos, resultando na diminuição da competição pela maior abertura do dossel e disponibilidade de nutrientes, permitindo maior crescimento dos indivíduos remanescentes e, ou, poderia também estar relacionada à ocorrência de espécies de estágios iniciais de sucessão, que possuem rápido crescimento. Van den Berg (2001) também encontrou maior ganho em área basal nas parcelas que apresentaram maior intensidade de perturbação e densidade inicial, o que foi atribuído à composição, nessas áreas, por espécies iniciais de rápido crescimento.

$\mathrm{O}$ crescimento em área basal das sobreviventes, positivo em todos os fragmentos em 2005-2007, foi negativo na floresta ciliar no período 2007-2009. Isso ocorreu, principalmente, em razão da grande mortalidade de perfilhos (bifurcações do caule) neste fragmento. Os perfilhos são comuns em árvores de florestas inundáveis e se apresentam como uma importante estratégia de adaptação aos ambientes com excesso hídrico, pois proporcionam aos indivíduos maior capacidade de sobrevivência por diminuir as chances de morte, permitindo alta capacidade de rebrota após as cheias e, assim, implicando em elevada capacidade de recuperação após enchentes (SILVA et al., 2011b). Além disso, houve também baixo crescimento dos sobreviventes (com exceção do Fragmento 3), que, segundo Guimarães et al. (2008), ocorre frequentemente em florestas aluviais, em razão do excesso hídrico, que pode ser causado pelo ambiente hipóxico ou anóxico que ocorre durante as enchentes.

\section{CONCLUSÕES}

Nos dois períodos avaliados, 2005-2007 e 20072009, o presente estudo demonstrou instabilidade da dinâmica florestal, com redução do número de indivíduos arbóreos e perda de área basal.

Cerne, Lavras, v. 17, n. 4, p. 465-471, out./dez. 2011
As hipóteses testadas de que as taxas de dinâmica apresentam variações no espaço e entre períodos avaliados foram aceitas. Diferenças da saturação hídrica do solo, controladas pelos eventos de enchentes, e do histórico de perturbação antrópica, podem explicar os resultados encontrados.

O monitoramento da comunidade arbórea será necessário para melhor conhecimento dos padrões de dinâmica.

\section{REFERÊNCIAS}

ANGIOSPERM PHYLOGENY GROUP. An update of the Angiosperm Phylogeny Group classification for the orders and families of flowering plants: APG III. Botanical Journal of

the Linnean Society, London, v. 161, n. 2, p. 105-121, 2009.

APPOLINÁRIO, V.; OLIVEIRA FILHO, A. T.;

GUILHERME, F. A. G. Tree population and community

dynamics in a Brazilian tropical semideciduous forest. Revista

Brasileira de Botânica, São Paulo, v. 28, n. 2, p. 347-360, 2005.

BRASIL. Ministério da Agricultura e Reforma Agrária.

Normais climatológicas 1961-1990. Brasília, 1992. 84 p.

CHAMBERS, J. Q.; HIGUCHI, N.; SCHIMEL, J. P. Ancient trees in Amazonia. Nature, London, v. 391, p. 135-136, 1998.

CONDIT, R. et al. Beta-diversity in tropical forest trees.

Science, New York, v. 295, n. 5555, p. 666-669, 2002.

DAMASCENO JÚNIOR, G. A. et al. Tree mortality in a riparian forest at Rio Paraguai, Pantanal, Brazil, after an extreme flooding. Acta Botanica Brasílica, Porto Alegre, v. 18, n. 4, p. 839-846, 2004.

\section{EMPRESA BRASILEIRA DE PESQUISA}

AGROPECUÁRIA. Sistema brasileiro de classificação de solos. Rio de Janeiro, 1999. 306 p.

FRANKLIN, J. F.; SHUGART, H. H.; HARMON, M. E. Tree death as an ecological process. BioScience, New York, v. 37, n. 8, p. 550-556, 1987.

GUILHERME, F. A. G. et al. Effects of flooding regimes and woody bamboos on tree community dynamics in a section of tropical semideciduous forest in South-Eastern Brazil. Plant Ecology, Dordrecht, v. 174, n. 1, p. 19-36, 2004. 
GUIMARÃES, J. C. C. et al. Dinâmica do componente arbustivo-arbóreo de uma floresta de galeria aluvial no planalto de Poços de Caldas, MG, Brasil. Revista Brasileira de Botânica, São Paulo, v. 31, n. 4, p. 621-632, 2008.

HIGUCHI, P. et al. Spatio-temporal patterns of tree community dynamics in a tropical forest fragment in Southeast Brazil. Plant Ecology, Dordrecht, v. 199, n. 1, p. 125135, 2008.

\section{INSTITUTO BRASILEIRO DE GEOGRAFIA E} ESTATÍSTICA. Manual técnico da vegetação brasileira.

Rio de Janeiro: Fundação IBGE, 1992. 92 p. (Série Manuais técnicos em geociências, 1).

JUNK, W. J. Wetlands of tropical South America. In: WHIGHAM, D.; HEJNÝ, S.; DYKYJOVÁ, D. (Ed.).Wetlands of the world I: inventory, ecology and management. Boston: W. Junk, 1993. p. 679-739.

KORNING, J.; BALSLEV, H. Growth and mortality of trees in Amazonian tropical rain forest in Ecuador. Journal of Vegetation Science, Ottawa, v. 4, n. 1, p. 77-86, 1994.

LIEBERMAN, D. et al. Mortality patterns and stand turnover rates in a wet tropical forest in Costa Rica. Journal of Ecology, Oxford, v. 73, n. 3, p. 915-924, 1985.

OLIVEIRA FILHO, A. T. et al. Dinâmica da comunidade e populações arbóreas da borda e interior de um remanescente florestal na Serra da Mantiqueira, Minas Gerais, em um intervalo de cinco anos: 1999-2004. Revista Brasileira de Botanica, São Paulo, v. 30, n. 1, p. 149-161, 2007.

OLIVEIRA FILHO, A. T.; MELLO, J. M.; SCOLFORO, J. R. Effects of past disturbance and edges on tree community structure and dynamics within a fragment of tropical semideciduous forest in south-eastern Brazil over a five years period: 1987-1992. Plant Ecology, Oxford, v. 131, n. 1, p. 45-66, 1997.
R DEVELOPMENT CORE TEAM. R: a language and environment for statistical computing. Vienna: R Foundation for Statistical Computing, 2009. Disponível em: <http:// www.R-project.org>. Acesso em: 16 out. 2009.

SHEIL, D.; JENNINGS, S.; SAVILL, P. Long-term permanent plot observations of vegetation dynamics in Bundongo, a Ugandan rain forest. Journal of Tropical Ecology, Champaign, v. 16, n. 6, p. 675-800, 2000.

SHEIL, D.; MAY, R. M. Mortality and recruitment rate evaluations in heterogeneous tropical forests. Journal of Ecology, Oxford, v. 84, n. 1, p. 91-100, 1996.

SILVA, A. C. et al. Dinâmica de uma comunidade arbórea após enchente em fragmentos florestais no sul de Minas Gerais. Revista Árvore, Viçosa, v. 35, n. 4, p. 883-893, 2011 a.

SILVA, A. C. et al. Florestas inundáveis: ecologia, florística e evolução. Lavras: UFLA, 2011b. 144 p.

SILVA, A. C. et al. Florística e estrutura da comunidade arbórea em fragmentos de Floresta Aluvial em São Sebastião da Bela Vista, Minas Gerais. Revista Brasileira de Botânica, São Paulo, v. 32, n. 2, p. 283-297, 2009.

SILVA, A. C.; HIGUCHI, P.; VAN DEN BERG, E. Effects of soil water table regime on tree community species richness and structure of alluvial forest fragments in South-East Brazil. Brazilian Journal of Biology, São Paulo, v. 70, n. 3, p. 15-21, 2011c.

SWAINE, M. D.; LIEBERMAN, D.; PUTZ, F. E. The dynamics of tree populations in tropical forest: a review. Journal of Tropical Ecology, Dordrecht, v. 3, n. 4, p. 359-366, 1987.

VAN DEN BERG, E. Variáveis ambientais e a dinâmica estrutural de populacional de uma floresta de galeria em Itutinga, MG. 2001. 115 p. Tese (Doutorado em Biologia Vegetal) - Universidade Estadual de Campinas, Campinas, 2001. 
